\title{
Feynman, a superfluidez e a supercondutividade
}

\section{Feynman, the superfluidity and the superconductivity}

\author{
Paulo F. Farinas*1 \\ ${ }^{1}$ Universidade Federal de São Carlos, Departamento de Física, São Carlos, SP, Brasil
}

Recebido em 21 de Janeiro, 2018. Revisado em 16 de Feveiro, 2018. Aceito em 20 de Fevereiro, 2018.

\begin{abstract}
É apresentada uma exposição breve e, na medida do possível, leve, das ideias em torno do entendimento da superfluidez e sua relação com a supercondutividade, com destaque ao papel de Richard Feynman. Tenta-se focalizar sobre o conteúdo das ideias em vez dos apectos sociais e históricos, cuja abordagem já popula a literatura. Palavras-chave: superfluidez, supercondutividade, Feynman.
\end{abstract}

A brief display of the ideas leading to the understanding of superfluidity, and its relation to superconductivity, is attempted, with focus on the role of Richard Feynman. Emphasis is tried to be put on the physical aspects, rather than on the sociological and historical ones, as the latter are extensively covered in the literature already. Keywords: superfluidity, superconductivity, Feynman.

\section{Introdução}

Em seu artigo publicado em número especial da Physics Today no ano de 1989 [1], D. Pines descreve que entre os cerca de 14 artigos científicos publicados por Feynman nos anos de 1953 a 1958, dois relacionam-se com desenvolvimentos de seu trabalho anterior em eletrodinâmica quântica, sendo os outros 12 em física da matéria condensada. Destes últimos, 10 tratam do hélio líquido, um aborda a relação entre supercondutividade e superfluidez e um lida com elétrons em cristais polares, cujas excitações elementares constituem os hoje conhecidos pólarons, tema de artigo nesta seção especial [2].

Este artigo lida com parte dessas contribuições relacionadas com os fenômenos da superfluidez. Exposições relativamente didáticas de parte das ideias abordadas aqui podem ser encontradas nos livros de Feynman [3], de mecânica estatística, e na parte 2 de mecânica estatística do livro da coleção de Landau e Lifshitz [4].

Ao escrever sobre o conteúdo físico dos trabalhos que deram um impulso tão diferenciado para o entendimento de questões fundamentais, fica claro, da releitura de parte desses trabalhos, que a generalidade e a elegância dessas abordagens são imensas. Portanto, é apresentado aqui o que poderia ser visto como uma tentativa de, décadas depois, reproduzir parte desses argumentos presumindo uma formação mais condizente com o tempo e o espaço a que estão ligados os leitores da Revista Brasileira de Ensino de Física. Presume-se ser uma formação mais completa em sua linguagem, mas possivelmente não familiarizada (em sua média) com argumentos tão gerais e elegantes. Acredito que esta não é uma característica

*Endereço de correspondência: farinas@df.ufscar.br exclusiva do estudante brasileiro de física, que hoje já vem sendo exposto ao mesmo conteúdo abordado em cursos de física mais tradicionais pelo mundo afora. Uma peculiaridade local está, é claro, na língua adotada para este artigo.

Pensando um pouco, é possível que Feynman apreciasse uma homenagem a ele que tenta focalizar sobre o conteúdo das ideias, reiterado na língua de uma cultura que o próprio Feynman experimentou durante um período de sua vida [5]. Isto é tentado nas próximas seções.

\section{Hélio 4}

O hélio foi "descoberto no Sol" antes de ser encontrado na Terra. Sua descoberta, feita indiretamente pela observação das linhas espectrais da radiação solar, engendrou o seu nome, cuja raiz grega remete à palavra que em grego significa "Sol". A observação ocorreu no início da segunda metade do século XIX e sua detecção na Terra cerca de dez anos mais tarde. A história do elemento é bastante rica, em particular no que concerne aos aspectos básicos do conhecimento que o seu estudo avançou, parte desses aspectos abordamos aqui. A Ref. [8] aborda a descoberta da superfluidez do ${ }^{4} \mathrm{He}$ e seus desenvolvimentos acadêmicos. Um resumo da história mais geral, com seus desdobramentos práticos e usos do elemento pode ser lido na Ref. 9].

Dos nove isótopos hoje conhecidos do hélio, dois são estáveis, ${ }^{4} \mathrm{He}$ e ${ }^{3} \mathrm{He}$, sendo o segundo muito menos abundante na Terra (por exemplo, estima-se que há na atmosfera cerca de 1 parte de ${ }^{3} \mathrm{He}$ para $10^{6}$ partes de ${ }^{4} \mathrm{He}$ ). 
O spin desses átomos (denominando "spin" a soma dos valores das projeções dos spins, sobre um eixo comum, dos prótons, nêutrons e elétrons que formam o átomo) resulta inteiro para o primeiro e semi-inteiro para o segundo. Esta propriedade de ter spin líquido inteiro ou semi-inteiro diferencia partículas da natureza nas duas classes observadas, chamadas de bósons e férmions em homenagem a dois grandes físicos que tiveram atuação direta no estabelecimento desses conceitos (S. N. Bose 10 e E. Fermi [1]). O que diferencia partículas classificadas como férmions e bósons é basicamente que os férmions, quando em conjunto, nunca partilham os números que caracterizam completamente os seus estados, já os bósons podem ter números iguais. Em outras palavras, dado um único nível completamente especificado pela energia (e possivelmente outros números quânticos), vários bósons podem ocupar este nível, enquanto apenas um férmion estaria presente em um nível desse tipo. Átomos têm extensão e movimentos internos, mas podemos tratálos como partículas ao estudarmos sistemas em que os átomos experimentam processos que envolvem trocas de energias muito pequenas comparadas com, por exemplo, a energia de ionização do átomo. É o caso dos átomos do hélio no estado líquido, por exemplo, que abordamos aqui, que se torna líquido a temperaturas bem baixas, da ordem de poucos K. A fase superfluida apenas se estabelece quando a temperatura é abaixada para pouco mais do que $2 \mathrm{~K}$ para o ${ }^{4} \mathrm{He}$ e pouco acima de $2 \mathrm{mK}$ para o ${ }^{3} \mathrm{He}$.
Em 1908, H. K. Onnes (que depois descobriu a supercondutividade em 1911) liquefez o hélio, abaixando sua temperatura para menos de $1 \mathrm{~K}$ à pressão ambiente. Em trabalhos publicados em 1927 e 1932 [12,13], observou-se que a fase líquida do ${ }^{4} \mathrm{He}$ tinha comportamentos distintos quando se cruza uma linha de temperatura que hoje se sabe ser relativamente independente da pressão, conforme mostra a Fig.1 em um diagrama de fases mais contemporâneo. Isto originou a nomenclatura He I e He II, esta última tendo sido reconhecida como a fase superfluida em 1938, nos trabalhos de Kapitza, e independentemente, Allen e Meissner, que foram publicados lado a lado na mesma edição da Nature 14, 15].

Para uma abordagem histórica da descoberta da superfluidez, recomendamos, entre outras presentes aqui que são oportunamente citadas, a Ref. [16].

\subsection{Superfluidez}

A situação do ${ }^{4}$ He no início da década de 1950 era a de um fluido conhecido, em que se descobriu uma transição superfluida 14, 15] cujo entendimento ainda estava em desenvolvimento, mas já havia avançado bastante. As duas fases chamadas de He I (fase normal) e He II (fase superfluida) já eram conhecidas. Um artigo de revisão de 1952 intitulado "Theories of He II" dá uma ideia do conhecimento da época [17].

Como o núcleo do ${ }^{4} \mathrm{He}$ tem spin total inteiro, seu comportamento em baixas energias (energias que não são altas o suficiente para alterar o valor do spin) segue a

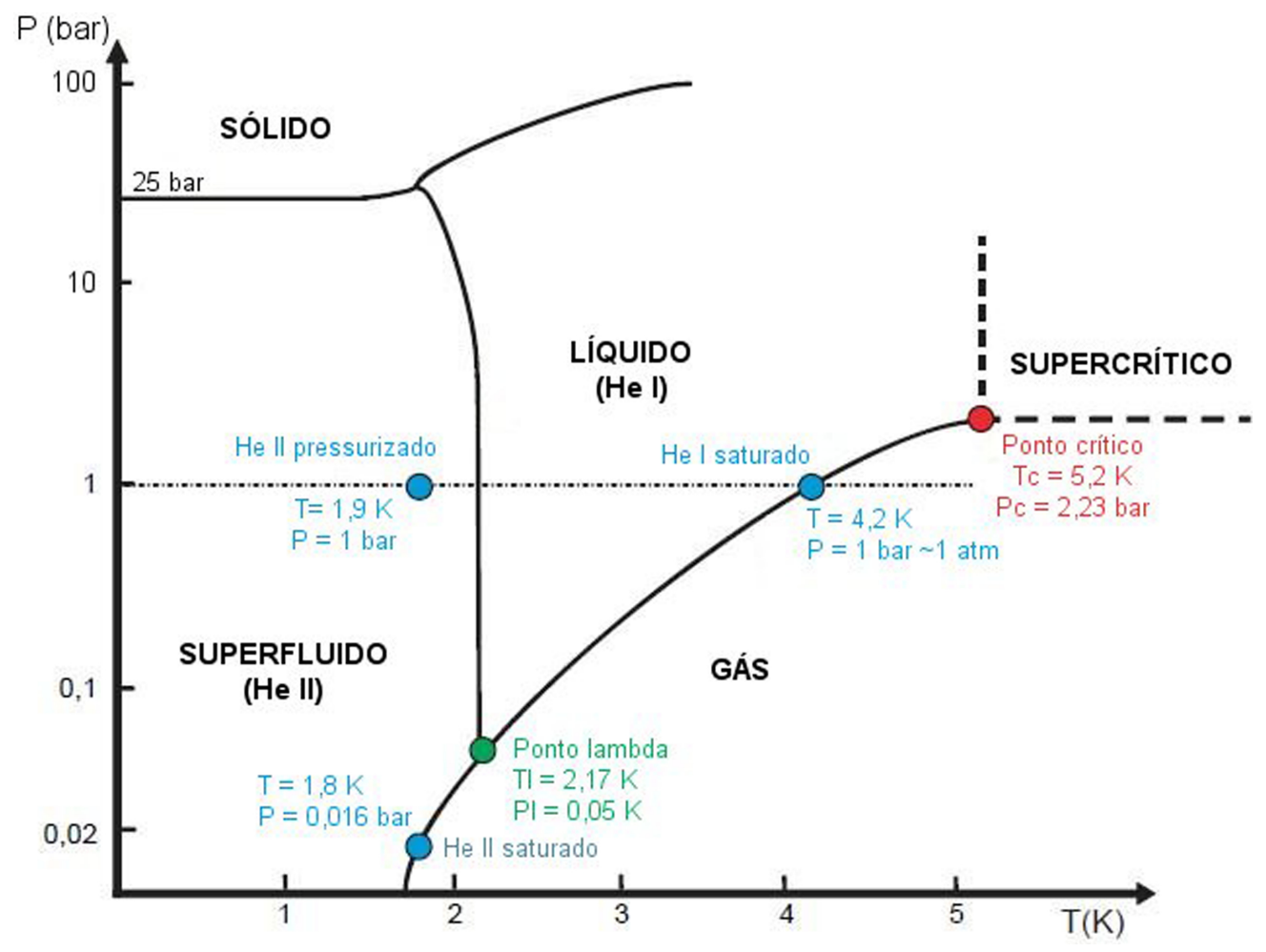

Figura 1: Diagrama de fases do ${ }^{4} \mathrm{He}$ como se conhece hoje. 
estatística bosônica (contrariamente ao isótopo parente ${ }^{3} \mathrm{He}$, que é um férmion).

A superfluidez de um líquido pode ser entendida como um estado em que todas as velocidades das partículas do líquido tendem a ser iguais, para valores de velocidade abaixo de um certo valor crítico acima do qual este estado correlacionado torna-se instável. Isto pode ser imaginado como possível, inicialmente de forma bem qualitativa, pensando na mecânica quântica do sistema, como segue. Para que tenham as mesmas velocidades em um estado, e que isto seja robusto, deve existir um nível quântico de energia isolado, com velocidade bem definida, e que permita a presença de várias partículas acomodadas (ou "presas") nesse nível. "Presas" aqui significa que o nível tem a menor energia possível entre todas (é o estado fundamental), e que a distância em energia até o próximo nível, com energia mais alta, é suficiente para que a temperatura não possa promover muitas partículas para o nível mais alto (isto requer temperaturas baixas). Com esse cenário, que a mecânica quântica permite, podemos imaginar todas as partículas do líquido, abaixo de uma certa temperatura, "trancadas" em um nível de energia com velocidades idênticas. Portanto, não apenas a temperatura não é suficiente para alterar a velocidade do fluido, mas se houver eventuais impurezas e/ou interações de partículas do líquido com, digamos, as paredes do capilar pelo qual ele flui, essas interações têm que ser intensas o suficiente para que a troca de energia resultante do espalhamento promova alguns dos componentes do líquido para estados de velocidade diferente. Este argumento é entendido mais diretamente se considerarmos a invariância de Galileu, que é uma invariância (muito bem) aproximada que deve existir entre referenciais inerciais para partículas com velocidades bem abaixo da velocidade da luz. No referencial em que o fluido está parado, as paredes do capilar movem-se com velocidade constante. Neste referencial, é o movimento das paredes que "tenta", pela interação delas com o líquido, acelerar este último para um estado de velocidade não nula. A física descrita neste referencial não deve ser diferente da descrita no referencial original, em que as paredes permanecem estacionárias. Essas interações com as paredes (ou impurezas) devem transferir energia e momento para o líquido pelo surgimento de excitações elementares do sistema, isto é, da promoção de partículas (ou "quase-partículas") para estados de energia mais alta. As características do espectro ditam o modo como as trocas de energia podem ou não ocorrer.

É importante salientar que o uso da invariância de Galileu aqui para ilustrar o argumento não é essencial. A conclusão geral é importante para o entendimento de muitos sistemas físicos. Entre eles, há um exemplo interessante ligado à área de tribologia: a observação de que o atrito entre duas superfícies, uma das quais pertencente a um material supercondutor, cai notavelmente ao, diminuindo-se a temperatura, cruzar a temperatura em que o estado supercondutor se estabelece.
Este fenômeno está ligado com a abertura de um gap de energia e a fomação de um estado correlacionado (um condensado) dificultando o espalhamento pela excitação de quase partículas. A ideia é análoga ao que acontece no superfluido, em que a interação com a parede do capilar não é suficiente para frear o líquido. Citamos a Ref. 18 como ponto de partida para uma literatura mais ampla, e modelos confirmando ou diminuindo o papel desta analogia na explicação dos detalhes que são observados nos diversos experimentos. Fenômenos como este são manifestações diretas do modo como estados de movimento são quantizados, e podem ser interpretados como observações macroscópicas da mecânica quântica.

A conclusão sugere que um sistema quântico, em seu estado fundamental, precisa de excitações elementares (envolvendo a dinâmica de estados excitados no espectro de energia) para que seu estado de movimento possa mudar. Neste caso tanto a mudança no sentido de acelerar quanto de frear o líquido (esta última mais comumente imaginada, pelo decaimento natural resultante da interação viscosa com as paredes de um capilar) estão relacionadas, por invariância de referenciais, com o mesmo fenômeno microscópico. Se não há possibilidade de espalhamentos bem sucedidos, por não haver energia suficiente para excitar esses modos, o sistema continua no seu estado original de movimento. Basicamente, este é o fenômeno (tirante diferenças importantes nos detalhes) que está por trás da supercondutividade e dos estados de resistência nula do efeito Hall quântico, por exemplo. Possivelmente o uso de um argumento análogo a este, em outro contexto, é mais familiar ao leitor, que é o fato de que bandas de energia cheias não permitem que elétrons conduzam eletricidade. Neste caso os elétrons são férmions, mas a conclusão baseia-se no fato análogo de que não há estados disponíveis para imprimir energia aos elétrons, a não ser na próxima banda, vazia e energeticamente difícil de acessar, para isolantes, em que o gap de energia é relativamente grande.

O modelo não interagente do condensado de BoseEinstein, já conhecido na época em que o entendimento da superfluidez no ${ }^{4} \mathrm{He}$ ainda estava no início, tem ingredientes que sugerem um estado superfluido. Por exemplo, há uma temperatura crítica abaixo da qual todos os seus componentes estão em um único nível de energia (o estado fundamental). O trabalho pioneiro de London [19], em 1938, relaciona o fenômeno de superfluidez no ${ }^{4} \mathrm{He}$, cujos átomos são bósons, com a teoria do condensado de Bose-Einstein de bósons não interagentes ("gás de BoseEinstein"). A ideia de London foi complementada pela teoria desenvolvida por Tisza [20], com um modelo de dois fluidos (a uma temperatura entre zero e a temperatura de transição, pode-se representar o líquido como uma combinação de duas densidades, uma normal, constituída pelas excitações elementares (ou quase-partículas), que carregam toda a entropia do líquido, outra superfluida, com entropia nula e associada às demais partículas, no estado condensado). 
Entretanto, se prosseguirmos para refinar o argumento qualitativo sobre o espectro de excitações elementares que demos acima, que se deve a Landau [4], usando a invariância de Galileu para ilustrar o argumento, vamos chegar à importante conclusão de que um sistema nãointeragente, como é o gás de Bose-Einstein (ou mesmo um sistema interagente que mantenha uma dispersão quadrática a baixas energias), não pode sustentar de modo estável um escoamento superfluido. Vejamos como isto segue.

A energia do fluido com todas as partículas à mesma velocidade $\overrightarrow{\boldsymbol{v}}$, cujo módulo indicamos por $v$, é dada por

$$
E_{0}=\frac{1}{2} M v^{2}+U=\frac{1}{2 M} P_{0}^{2}+U
$$

onde $M$ é a massa do fluido, $\overrightarrow{\boldsymbol{P}}_{0}$ seu momento, e $U$ dá conta de todos os termos não-cinéticos da energia. Ao interagir, digamos, com as paredes do tubo capilar, o surgimento de uma única excitação elementar com momento $\overrightarrow{\boldsymbol{p}}$ e energia $\varepsilon(\overrightarrow{\boldsymbol{p}})$ no fluido muda a sua energia para

$$
E_{F}=\frac{1}{2 M}\left(\overrightarrow{\boldsymbol{P}}_{0}+\overrightarrow{\boldsymbol{p}}\right)^{2}+U+\varepsilon(\overrightarrow{\boldsymbol{p}})=E_{0}+\overrightarrow{\boldsymbol{p}} \cdot \overrightarrow{\boldsymbol{v}}+\varepsilon(\overrightarrow{\boldsymbol{p}}),
$$

ou

$$
\Delta E=E_{F}-E_{0}=\overrightarrow{\boldsymbol{p}} \cdot \overrightarrow{\boldsymbol{v}}+\varepsilon(\overrightarrow{\boldsymbol{p}}),
$$

diferença resultante da transferência de momento e energia para o sistema, suficientes para promover uma única excitação. Note que o termo $p^{2} / 2 M$ foi ignorado na expressão acima. Podemos justificar isto argumentando que o momento $\overrightarrow{\boldsymbol{p}}$ de uma única excitação elementar deve ter seu módulo da ordem de $p \sim P_{0} / N$, onde $N$ é o número de partículas do líquido e $P_{0}$ o módulo do momento total do líquido. Portanto,

$$
|\overrightarrow{\boldsymbol{p}} \cdot \overrightarrow{\boldsymbol{v}}|=\left|\overrightarrow{\boldsymbol{p}} \cdot \frac{\overrightarrow{\boldsymbol{P}}_{0}}{M}\right| \sim \frac{1}{N} \frac{P_{0}^{2}}{M},
$$

enquanto

$$
\frac{p^{2}}{2 M} \sim \frac{1}{2 N^{2}} \frac{P_{0}^{2}}{M} .
$$

Portanto, no limite termodinâmico $(N \rightarrow \infty$ e o volume $V \rightarrow \infty$, com a densidade $N / V$ convergindo) desprezar o termo quadrático em $p$ leva a um resultado exato.

A diferença de energia deve ser negativa se a excitação elementar contribui para frear o líquido. Portanto, a condição para que exista superfluidez é que a diferença seja positiva ou nula, isto é, $\overrightarrow{\boldsymbol{p}} \cdot \overrightarrow{\boldsymbol{v}}+\varepsilon(\overrightarrow{\boldsymbol{p}}) \geq 0$. O menor valor de $\overrightarrow{\boldsymbol{p}} \cdot \overrightarrow{\boldsymbol{v}}$ ocorre para $\overrightarrow{\boldsymbol{p}}$ e $\overrightarrow{\boldsymbol{v}}$ antiparalelos, logo a condição pode ser posta em termos apenas dos módulos, $-p v+\varepsilon(p) \geq 0$ e rescrita como

$$
v \leq \frac{\varepsilon(p)}{p}
$$

Esta condição informa que para velocidades abaixo de um certo valor, não é possível provocar excitações com um certo par momento-energia no líquido.
A relação (2) é apenas uma reafirmação quantitativa acerca das hipóteses que usamos para definir a superfluidez. A impossibilidade de excitações elementares por espalhamento (interação com fontes externas, como as paredes de um capilar) e a presença de um estado de velocidade única ocupado por todas as partículas foram impostas sobre o balanço de energia levando a esta relação.

Ela contém uma informação importante sobre a forma da relação de dispersão $\varepsilon(p)$ das próprias excitações elementares. Essa relação deve satisfazer a desigualdade oposta para que existam excitações, isto é,

$$
\frac{\varepsilon(p)}{p} \leq v
$$

onde $v$ é o módulo da velocidade do superfluido. Para que haja esta limitação, a curva do espectro de excitações de baixa energia tem que se aproximar de zero de forma linear. Apesar de um argumento gráfico ser possível, podemos notar isto mais diretamente analisando a expansão de $\varepsilon(p)$. Supondo a função como analítica, o que é razoável, temos

$$
\varepsilon(p)=\varepsilon_{0}+v_{1} p+b p^{2}+\ldots
$$

ou ainda

$$
\frac{\varepsilon(p)}{p}=\frac{\varepsilon_{0}}{p}+v_{1}+b p+\ldots
$$

Vemos que o primeiro termo diverge se $\varepsilon_{0} \neq 0$, e não pode satisfazer à condição (3), violando-a justamente quando $p$ se torna pequeno. Com isto, vemos que $\varepsilon_{0}=0$ e o espectro deve ser do tipo

$$
\varepsilon(p)=v_{1} p+b p^{2}+\ldots
$$

Mas isto ainda não exclui a possibilidade $v_{1}=0$, por exemplo, o que sugeriria que um espectro quadrático quando $p \rightarrow 0$ é consistente com estes argumentos. Note, porém, que se $v_{1}=0$, a condição (3) é satisfeita para velocidades arbitrariamente pequenas incluindo velocidade nula. Portanto, se $v_{1}=0$ podemos ter excitações para alguma faixa de valores de momentos arbitrariamente pequenos, para qualquer velocidade do fluido. A possibilidade de excitações elementares para qualquer velocidade, por menor que seja, indica que o movimento é sempre atenuado e a superfluidez não é estável.

Em suma, para que satisfaça à desigualdade derivada das condições para existência de superfluidez, o espectro de excitações elementares de um líquido deve ser linear a baixas energias. Na matéria, este é o espectro que emerge para fônons longitudinais (ou "acústicos"). As excitações de mais baixa energia de um tal sistema são os quanta de modos acústicos longitudinais. Isto exclui imediatamente qualquer sistema com espectro quadrático, como é o caso do gás de bósons. Portanto, apesar de possuir ingredientes essenciais para o entendimento de condensados fracamente interagentes, o modelo de gás 
de bósons não é adequado para entender a superfluidez do ${ }^{4} \mathrm{He}$.

$\mathrm{O}$ argumento que propõe o espectro foi feito de modo mais sucinto e elegante por Landau em um trabalho 21. e sua complementação 22 fitando resultados experimentais. Esses trabalhos fazem parte de um esforço que veio influenciar a menção explícita ao hélio na justificava dada pelo comitê do Nobel de Física ao escolher Landau como único recipiente do prêmio no ano de 1962.

No que se refere ao espectro, o enfoque do trabalho original 21] não é como o dado aqui (a exposição acima é mais alinhada com a que se encontra no livro de sua coleção 4]). A argumentação original baseia-se no que Feynman, por exemplo, chamou de "quantização da hidrodinâmica". Nele, Landau constrói operadores quânticos para quantidades típicas da dinâmica clássica de um fluido homogêneo, como a densidade $\rho$ e a circulação do campo de velocidades $\boldsymbol{\nabla} \times \boldsymbol{v}$, e avalia as relações de comutação entre esses observáveis (e entre suas componentes, no caso de vetores). Referindo-se a uma analogia com os operadores de momento angular (que acreditamos que ele sabia não ser necessária, mas que encurta o caminho para o entendimento, numa época em que mesmo a quantização do momento angular ainda era recente), ele conlcui que o espectro, quantizado, deve ter um estado fundamental isolado com circulação nula, portanto com escoamento potencial. Da mesma forma que as relações de comutação para o momento angular implicam na existência de um espectro discreto com valores bem definidos, a circulação satisfaz a relações de comutação análogas às do momento angular, tendo portanto um espectro discreto. Rigorosamente, a conclusão lógica dos seus argumentos não implica que o estado com circulação nula (escoamento potencial) é o de menor energia, ela apenas indica que existem estados com circulação nula e outros com circulação não nula separados em um espectro discreto. Landau supôs que o estado de circulação nula seria o estado fundamental porque isto levava a uma explicação para a superfluidez. Um campo sem circulação é o que chamamos de campo longitudinal, um conceito que surge quando decompomos um campo vetorial na soma de um vetor transverso com um longitudinal (decomposição de Helmholtz). As componentes de Fourier, $\delta \boldsymbol{\psi} \sim \boldsymbol{A}_{\boldsymbol{k}} e^{i \boldsymbol{k} \cdot \boldsymbol{r}}$, de pequenas oscilações de um campo longitudinal são paralelas à direção de propagação $\hat{\boldsymbol{k}}$, já que $\boldsymbol{\nabla} \times \delta \boldsymbol{\psi}=0 \Longrightarrow \boldsymbol{k} \times \delta \boldsymbol{\psi}=0$. Portanto as perturbações de um campo longitudinal constituem modos longitudinais de compressão, cujos quanta são fônons acústicos, com dispersão linear. Campos longitudinais não podem sustentar outros tipos de oscilação já que, por definição, os vetores de onda de perturbações a esses campos são paralelos aos próprios campos. Este argumento permitiu que Landau derivasse de um modo muito geral o espectro de baixas energias. Além disso, ele pôde também avaliar as possíveis propriedades dos primeiros estados excitados, já que estes são estados com circulação não nula, o que leva à possibilidade de formação de vórtices para as ex- citações de energia mais alta (portanto presumivelmente mais comuns para temperaturas nas proximidades da transição). Isto se resume em um espectro com dois tipos de excitações elementares no superfluido: as de momento pequeno, constituídas de fônons acústicos (dispersão linear) e as de momento e energia mais altos que, por causa da possibilidade de associarem circulação não nula, em contraste com os fônons acústicos, foram chamadas de "rotons". A Fig. 2 mostra um gráfico do espectro previsto por Landau e os resultados experimentais que vieram confirmar a previsão alguns anos depois.

Parte das críticas à generalidade do trabalho de Landau baseava-se no papel obscuro da estatística de BoseEinstein em sua argumentação. Usava-se como uma certa "garantia empírica", ou um reforço ao argumento, o fato de que ${ }^{3}$ He não se tornava superfluido, sendo que a generalidade do argumento faz com que ele se estenda ao ${ }^{3} \mathrm{He}$ (com a diferença de que férmions têm excitações elementares que aparecem em pares, já que o momento angular de qualquer sistema quântico varia sempre em múltiplos de $\hbar$, e férmions têm spin semi-inteiro). Mais tarde, o ${ }^{3} \mathrm{He}$ revelou tornar-se superfluido a temperaturas tão mais baixas que a descoberta só viria a acontecer em 1972 [24].

Em maior ou menor dosagem, tais críticas podem ser encontradas reproduzidas em textos de Feynman 3, 25. Uma delas é a ausência de um cenário microscópico na teoria de Landau: qual o papel objetivo da estatística? Como é a função de onda do superfluido? Estas são perguntas que foram respondidas por Feynman, avançando o entendimento microscópico da superfluidez. Feynman pôde derivar as propriedades do superfluido fazendo argumentos sobre a amplitude da função de onda, com especial enfoque na simetria ditada pela estatística de

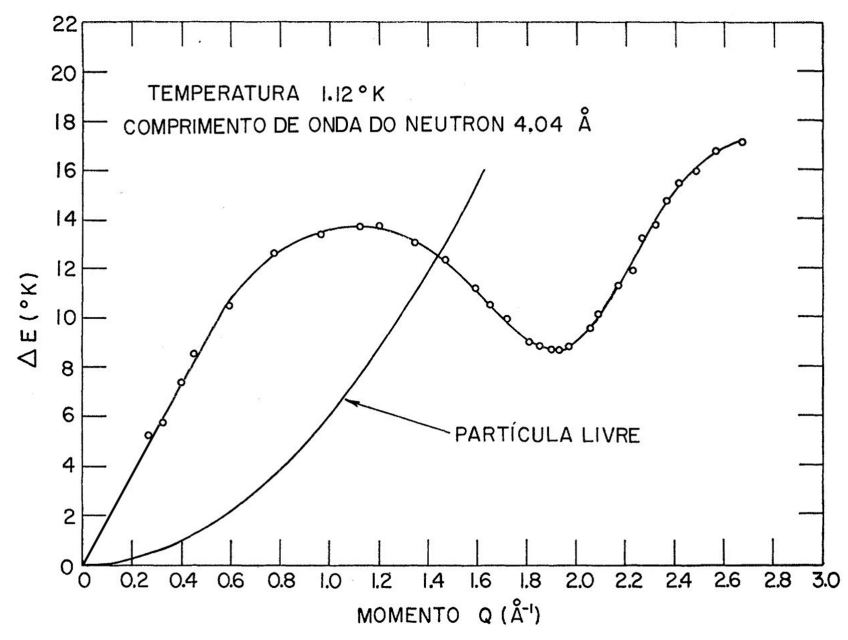

Figura 2: Espectro de excitações. do ${ }^{4} \mathrm{He}$ obtido por espalhamento de nêutrons 23]. O mínimo em momentos mais altos corresponde às excitações chamadas de rotons. Para momentos pequenos, o espectro é linear o que corresponde a excitações cujos quanta são fônons acústicos. A curva parabólica corresponde ao espectro de partículas não interagentes com a massa do ${ }^{4} \mathrm{He}$. 
Bose-Einstein, que impõe que a função não troque de sinal pela troca dos números que identificam duas partículas e também no papel da interação forte e de curto alcance entre os átomos de hélio.

\subsection{Detalhes microscópicos - Feynman}

Como vimos, a teoria de Landau é de uma generalidade muito ampla, e na época entendia-se que o papel da estatística bosônica ficava depreciado na abordagem dada por Landau. Isto gerou alguma disputa acadêmica entre as abordagens dadas por London e Tisza, que enfatizavam o papel da estatística de Bose-Einsten para a superfluidez, e a de Landau, que focalizava em argumentos mais gerais. Houve pelo menos um episódio, narrado na Ref. [26] que culminou com Landau e Tisza trocando "cartas para o editor" em tom bastante escolástico, mas em que Landau criticava toda a teoria de Tisza, e afirmava, em uma nota de rodapé que ela estava "inteiramente incorreta" 27,28. Em seu texto, Landau defende os aspectos de sua teoria que eram alvo de alguma crítica.

A contribuição de Feynman para o entendimento da superfluidez a baixas temperaturas foi mostrar que os conceitos mais gerais da teoria de Landau emergiam de fundamentos da mecânica quântica aplicada para um conjunto de bósons interagindo fortemente através de um potencial local. Na seção 11.3, página 321 da sétima tiragem de seu livro de mecânica estatística [3], ele argumenta de modo muito claro (que não seria mais que mera reprodução repetir aqui) e nos leva às propriedades essenciais da amplitude (função de onda) do estado fundamental, $\varphi\left(\boldsymbol{r}_{1}, \boldsymbol{r}_{2}, \ldots, \boldsymbol{r}_{N}\right)$ de um sistema de $N$ bósons interagentes, com posições denotadas pelas $3 \mathrm{~N}$ coordenadas $\boldsymbol{r}_{i}$ 's. Ele conclui por argumentos relativamente simples e hoje comuns em cursos de mecânica quântica, que o estado fundamental é não-degenerado, e que a sua amplitude $\varphi$ deve ser simétrica (por troca de posições), real, sempre positiva, grande quando a configuração é homogênea (átomos separados ao máximo) e pequena para configurações que têm átomos próximos ou com orbitais sobrepostos.

Os mesmos argumentos aplicados para o primeiro estado excitado, levam Feynman a concluir que sua amplitude $\psi$ tem as mesmas propriedades de $\varphi$ exceto ser sempre positiva. Para o primeiro estado excitado, $\psi$ deve ter um nodo (em relação à variação de uma única coordenada com as outras $3 N-1$ fixas). Como o primeiro estado excitado (amplitude $\psi$ ) deve ser ortogonal ao estado fundamental (amplitude $\varphi$ ), a integral de $\varphi \psi$ sobre todas as configurações deve ser igual a zero. Para isto, $\psi$ deve ser positiva para cerca de metade das configurações e negativa para as demais (pois $\varphi$ é positiva para todas as configurações). A contribuição de configurações tais que algumas poucas têm amplitude $\psi$ grande e positiva e as demais têm amplitude pequena e negativa, mas em maior número (o que matematicamente também zera a integral), são descartadas por terem derivadas espaciais maiores associadas a elas, como o argumento adiante esclarece estar em desacordo com a restrição imposta pelo critério de baixa energia.

Para demonstrar que o espectro de excitações de baixa energia só pode conter flutuações longitudinais de densidade, Feynman faz um argumento que pode ser construído se supomos que, em geral, as flutuações de densidade são, digamos, proporcionais a $|\psi|^{2}$, já que $\psi$ é a amplitude do primeiro estado excitado. A energia do estado fundamental, sendo a mais baixa, é escolhida como sendo igual a zero. Todos os estados excitados têm energia positiva, e estamos interessados no de mais baixa energia entre eles, que chamamos de primeiro, usando a energia como critério de ordenamento. Para que $\psi$ seja um autoestado de baixa energia, suas derivadas espaciais devem ser pequenas, o que se conclui simplesmente do modo como essas derivadas e a energia do autoestado de amplitude $\psi$ estão arranjadas na equação de Schrödinger do sistema de muitos corpos. Feynman analisa como essas derivadas mudam de valor, imaginando mudanças nas configurações dos átomos, isto é, imaginando variados conjuntos $\left\{\boldsymbol{r}_{1}, \ldots \boldsymbol{r}_{N}\right\}$, com possíveis trocas e permutações das posições que aparecem no argumento de $\psi$ e as consequentes variações produzidas em $\psi$. Quais trocas feitas nesse conjunto produzem as menores variações de $\psi$ ? Essas trocas de configuração que fazem $\psi$ variar são fortemente restringidas pela condição de homogeneidade (por causa do caroço de interação forte e repulsiva entre os átomos) e também pela estatística. Trocas em que os átomos movem-se pouco mas tendem a aproximar-se devem gerar grandes variações pela primeira condição (interação), e são descartadas, enquanto trocas que envolvem átomos distantes apenas permutando suas posições mantém $\psi$ inalterada. Sobram, como possíveis para contribuir com as flutuações da densidade, trocas entre átomos distantes mas com pequenas variações nas posições originais, de modo que átomos vizinhos também se rearranjem para manter o máximo de homogeneidade, evitando variações rápidas de amplitude por causa da interação forte, com caroço repulsivo, entre os átomos. Este tipo flutuação é satisfeito maximamente por vibrações longitudinais, e tanto mais satisfeito quanto maior fizermos o comprimento de onda (associado à escala de distância da variação das posições dos átomos). Espectros côncavos são, para momentos suficientemente pequenos, uma aproximação do caso parabólico (não interagente), isto é, sempre associam excitações de partícula livre para algum valor de momento suficientemente pequeno. Isto permite trocas com energias ainda menores, mas eles estão excluídos por permitirem trocas que, apesar de satisfazerem à restrição estatística, são indiferentes à importante restrição imposta pela interação.

Assim, Feynman consegue explicar qualitativamente, avançando um entendimento microscópico, de modo intuitivo, que o espectro deve ser linear (fônons acústicos) a baixas energias, como resultado das restrições básicas impostas pela estatística bosônica e pela interação forte 
entre os átomos. Feynman aprofunda sua análise, e obtém a partir dela, uma forma geral para a função de onda [3], e também para o espectro de baixas energias dada por

$$
\varepsilon(p) \sim \frac{p^{2}}{2 m S(p)},
$$

onde $S(p)$ é o fator de estrutura estático (que pode ser medido experimentalmente por espalhamento de nêutrons ou raios $\mathrm{X}$ ). Como

$$
S(p) \rightarrow \frac{p}{2 m c}
$$

para $p \rightarrow 0$, onde $c$ é a velocidade das ondas de compressão, o espectro é linear a baixas energias 29.

A cronologia dos trabalhos que levaram a este avanço, alguns deles publicados em sequência em volumes do Physical Review, inclui um primeiro artigo [30] onde ele trata a transição He I $\rightarrow$ He II, escrevendo a função de partição pelo uso da técnica de integrais de caminho inventada por ele [31], seguido de um trabalho onde ele explica a escassez de estados para um sistema interagente de bósons [32], e depois de um trabalho em que ele constrói os argumentos microscópicos para explicar o espectro inicialmente proposto por Landau em bases mais gerais [33], usando argumentos similares aos que esboçamos nos parágrafos acima.

A análise feita por Feynman estende-se, em colaboração com seu estudante na época, Michael Cohen 34 35] para o resto do espectro, com argumentos baseados em premissas fundamentais bastante parecidas com as expostas aqui.

Uma contribuição distinta que vem nesta sequência de trabalhos seminais é talvez, a julgar pela análise da literatura 36], considerada a mais importante delas. Em parte por ser mais geral, tendo sido fundamental também para o entendimento da supercondutividade 37]. Além de contribuir com a perspectiva diferenciada, em busca de detalhes microscópicos, ela aprofunda o entendimento de questões essenciais para o comportamento do superfluido a temperaturas mais altas, próximas da transição. Trata-se do seu trabalho de 1955 [38], em que ele estuda com detalhes a quantização da circulação, já visível nos trabalhos pioneiros de Landau, e explicitamente, mas sucintamente, sugerida por Onsager, em uma nota de rodapé de um artigo publicado em 1949 [39]. De acordo com J. R. Donnelly, a atribuição de importância científica à citação de Onsager sobre a quantização da circulação teria sido amplificada. Além da nota de rodapé referida acima, Lars Onsager expunha essa ideia para seus alunos e colegas na Universidade de Yale, a partir de 1946, e teria dito a seguinte frase, em uma palestra na "Conference on Statistical Mechanics" em Florença, em 1949, [40]

"...thus the well-known invariant called the hydrodynamic circulation is quantized; the quantum of circulation is $\hbar / m \ldots$ In the case of cylindrical symmetry, the angular momentum per particle is a multiple of $\hbar . "$
Ainda de acordo com Donnelly, na Ref. [40], "formaram-se ramificações enormes dessa única declaração e observouse mais de uma vez que a razão entre importância científica e o tamanho da anunciação deve ser um recorde na história da ciência".

Atribui-se a Feynman e Onsager, por esses trabalhos, a descoberta teórica da quantização dos vórtices. O entendimento da dinâmica desses objetos foi essencial para corrigir a previsão de Landau, de cerca de $60 \mathrm{~m} / \mathrm{s}$, para o valor crítico da velocidade do superfluido, um valor em torno de 100 vezes maior do que o medido. Foi a sugestão de Feynman, indicando como a formação de vórtices altera o valor da velocidade crítica, que permitiu a obtenção de valores teóricos mais de acordo com as observações experimentais. Para os leitores familiarizados com a supercondutividade mas não com o hélio, essa descrição pode parecer familiar. Na próxima seção descrevemos brevemente a influência dessas ideias sobre a supercondutividade.

Como fazer notória a importância de estudar-se o hélio líquido? Ou o fenômeno da superfluidez, incluindo a supercondutividade? E, mais geralmente, do estudo de excitações elementares na matéria? Para alguns, que entendem a envergadura das pessoas que foram atraídas para o tema, em particular a de Feynman, a quem esta série de artigos reverencia, bastariam as citações aqui presentes, ligadas a inúmeras a que não fizemos jus, mas que podem ser facilmente rastreadas. Mesmo para estes "bons entendedores", encerramos esta seção recomendando uma olhada no índice (e, quem sabe, a aventura de ler o conteúdo) da Ref. [41], que aponta para o livro de G. E. Volovik intitulado "O Universo em uma Gota de Hélio".

Antes de passar à próxima seção, cabe destacar que parte das ideias sobre a superfluidez foram concebidas por Feynman enquanto visitava o Centro Brasileiro de Pesquisas Físicas (CBPF), no Rio de Janeiro, e interagia com cientistas radicados no Brasil, entre eles José Leite Lopes, Jayme Tiomno, Walter Schutzer e Guido Beck. Feynman visitou o CBPF a convite de J. L. Lopes, durante 10 meses para um ano sabático, nos anos de 1951 e 1952, no então recém inaugurado prédio do CBPF. Ele conversou, também no Rio e na SBPC de julho de 53, com David Bohm, que na época estava na USP. A interação com estes cientistas era mais voltada para a física de partículas, mas durante sua estadia ele trabalhava também para entender a superfluidez. As Referências 6 (nesta seção especial da RBEF), [42 e 7 . narram partes da experiência de Feynman em sua interação com o Brasil. Vale destacar aqui um pequeno trecho desta narrativa [7]:

"By the summer of 1953, Feynman had already been thinking about the problem of the superfluidity of liquid helium for a couple of years almost constantly. During his stay in Rio de Janeiro in 1951-52, several ideas came to him concerning the solution of various aspects of this problem and he discussed them with Leite Lopes." 


\section{Supercondutividade}

A história da supercondutividade (que se mistura com a da superfluidez) aparentemente começa no início do século XIX com os esforços de Michael Faraday (o mesmo do eletromagnetismo) para liquefazer gases. Faraday foi empregado por Sir Humphry Davy, um conhecido químico inglês que atacava a (hoje universalmente conhecida) proposta do também inglês Dalton, das proporções múltiplas justificando a teoria atomística da matéria. Davy argumentava que 28, entre os cerca de 50 elementos conhecidos na época, eram metais, cujas propriedades, como brilho, boas condutividades térmica e elétrica, eram muito similares para serem emergentes da composição de 28 elementos distintos (hoje sabemos que os átomos têm estrutura interna, e que essas propriedades "similares" são devidas aos elétrons que constituem os "super orbitais" que os metais têm em comum ("elétrons livres") e que mediam as interações de baixa energia dos metais com o meio externo).

Faraday resolveu, como assistente de Davy, e tendo liquefeito um gás em uma das primeiras vezes em que isto foi conseguido, estabelecer-se a meta de liquefazer todos os gases conhecidos. Isto iniciou uma corrida em que vários nomes conhecidos da história da ciência tiveram alguma participação, e que levou o holandês Heike Kamerlingh Onnes a, em 1908, conseguir liquefazer o ${ }^{4} \mathrm{He}$, obtendo a menor temperatura já conseguida até então 43, um pouco abaixo de $1 \mathrm{~K}$, como já mencionamos aqui antes. Três anos depois, o próprio grupo de Onnes viria a descobrir a supercondutividade [44. Uma leitura agradável com detalhes dessa evolução, e também sobre o papel de Feynman no entendimento do fenômeno da supercondutividade, pode ser apreciada na Ref. [37]. Uma outra abordagem enfocando a história da liquefação dos gases e a descoberta de Onnes está na Ref. [45].

O problema da supercondutividade levou quase 50 anos para ser entendido completamente, tendo a teoria sido estabelecida por Bardeen, Cooper e Schrieffer [46]. London 47], Ginzburg e Landau 48], entre outros, avançaram o entendimento das propriedades mais gerais, com descrições fenomenológicas ou semi-fenomenológicas. A descoberta de W. Meissner e R. Ochsenfeld [49] do hoje conhecido efeito com o nome desses cientistas, mostrou que o supercondutor era mais do que um condutor perfeito, que congelaria as linhas de campo magnético em seu interior, sendo na verdade um diamagneto perfeito (as linhas são sempre excluídas do supercondutor, mesmo que haja campo magnético penetrando no material antes dele tornar-se supercondutor). Um material que pudesse tornar-se condutor perfeito, do ponto de vista do eletromagnetismo e da termodinâmica, teria as linhas de campo magnético "congeladas" em seu interior, pois um condutor perfeito não deixa linhas de campo surgirem ou sumirem (correntes de indução surgem em oposição às variações de campo e anulam a tendência de mudar o fluxo magnético). No caso do supercondutor, as linhas são sempre excluídas, elas não penetram no material, a não ser numa camada fina a partir de sua superfície. A escala de comprimento associada com a espessura desta camada é o comprimento de penetração de London.

Os supercondutores inicialmente conhecidos, a maioria metais puros, comportavam-se como diamagnetos perfeitos para campos magnéticos externos até um determinado valor crítico para o qual as linhas de campo penetram no material destruindo completamente sua supercondutividade, em uma transição de primeira ordem. Supercondutores desse tipo são chamados de supercondutores do tipo I. Ginzburg e Landau desenvolveram uma teoria fenomenológica para a supercondutividade em 1950, que hoje leva os seus nomes, que além de descrever as propriedades dos supercondutores do tipo I, previa a existência de supercondutores do tipo II [48]. Os próprios autores comentavam que não havia motivação experimental para considerar esses supercondutores "de segunda espécie" (todos os supercondutores conhecidos eram do tipo I). No entanto, os do tipo II já haviam sido observados experimentalmente por Rjabinin e Shubnikov em 1935 50, o que hoje os credencia pela descoberta. Os supercondutores do tipo II têm dois campos magnéticos críticos. O menor, quando é atingido por baixo, inicia um processo penetração de linhas de campo no material pela formação de vórtices que se constituem essencialmente de filetes com núcleo normal circundado por correntes supercondutoras. Essas estruturas podem aprisionar-se em impurezas e possuem uma dinâmica muito rica, muito estudada até hoje.

A teoria dos vórtices foi grandemente desenvolvida por Abrikosov [51], mas aparentemente houve um forte condicionante dado pelos trabalhos de Feynman [38] e Onsager [39] sobre a quantização de vórtices. O próprio Abrikosov narra em sua "Nobel Lecture" [52]:

..."I made my derivation of the vortex lattice in 1953 but publication was postponed since Landau at first disagreed with the whole idea. Only after R. Feynman published his paper on vortices in superfluid helium [38], and Landau accepted the idea of vortices, did he agree with my derivation, and I published my paper [51]."

Uma outra contribuição de Feynman para a supercondutividade tem relação com o tunelamento Josephson em supercondutores [53. Como muitas, a evolução da história deste efeito tem desdobramentos dramáticos interessantes, ligados à dificuldade que Josephson encontrou inicialmente para afixar sua ideia junto à comunidade científica, que contrasta com a rapidez (menos de dois anos) entre a previsão e sua verificação experimental. Deixaremos estes aspectos para que o leitor se divirta com a prospecção, caso seja do seu interesse. O modelo para o tunelamento que é discutido por Feynman aparentemente não deu origem, ele mesmo, a nenhum artigo científico assinado por Feynman, ele está publicado em um de seus textos didáticos mais básicos, sua famosa 
coleção ou suas "Lectures" [54]. Estas foram inspiradas em palestras dadas por Feynman, depois imortalizadas na coleção (que hoje tem tradução para o português), e tiveram início com uma série de apresentações para os alunos ("lectures") do Caltech em 1964.

Um registro mais detalhado é feito na Ref. [37, tanto dessas apresentações e a reação de Feynman ao efeito Josephson, quanto do testemunho dado por cientistas que contribuíram para o entendimento da supercondutividade, do papel importante de Feynman, quando não contribuindo diretamente, tendo sido um dos pivôs da competição em busca de uma explicação para o entendimento do fenômeno.

Com relação ao modelo de Feynman para o tunelamento Josephson, mesmo não tendo sido publicado em um artigo científico, o modelo é bastante utilizado em artigos científicos para o estudo de junções Josephson, até os dias de hoje.

\section{Agradecimentos}

Agradeço ao Professor Nelson Studart pela leitura crítica deste texto, e pelas inúmeras dicas dadas tanto antes quanto depois da leitura.

\section{Referências}

[1] D. Pines, Physics Today 42, 61 (1989).

[2] N. Studart, Rev. Bras. Ens. Fis 40 (2018). unpublished.

[3] R.P. Feynman, Statistical Mechanics - A Set of Lectures (Benjamin-Cummings, Reading, 1982).

[4] L.D. Landau and E.M. Lifshitz, Statistical Physics (Pergamon Press, Oxford, 1981), v. 9, part 2.

[5] R.P. Feynman, O Senhor Está Brincando, Sr. Feynman! (Elsevier, Rio de Janeiro, 2006).

[6] I.C. Moreira, Rev. Bras. Ens. Fis 40 (2018). unpublished.

[7] Jagdish Mehra, The Beat of a Different Drum - Life and Science of R.Feynman (Oxford University Press, Oxford, 1996).

[8] J.R. Donnelly, Phys. Today 48, 30 (1995).

[9] Wikipedia, disponível em https://en.wikipedia.org/ wiki/Helium.

[10] Wikipedia, disponível em https://en.wikipedia.org/ wiki/Satyendra_Nath_Bose

[11] Wikipedia, disponível em https://en.wikipedia.org/ wiki/Enrico_Fermi.

[12] M. Wolfke and W.H. Keesom, Proc. Amst. 31, 81 (1927).

[13] W.H. Keesom and K. Clusius, Leiden Comm. 219e (1932).

[14] P. Kapitza, Nature 141, 74 (1938).

[15] J.F. Allen and A.D. Meissner, Nature 141, 75 (1938).

[16] S. Balibar, J. Low Temp. Phys. 146, 441 (2007).

[17] R.B. Dingle, Adv. Phys. 1, 111 (1952).

[18] A. Dayo, W. Alnasrallah and J. Krim, Phys. Rev. Lett. 80, 1690 (1998).

[19] F. London, Phys. Rev. 54, 947 (1938).

[20] L. Tisza, Nature 141, 913 (1938).

[21] L. Landau Phys. Rev. 60, 356 (1941).
[22] L. Landau, J. Phys. U.S.S.R. 11, 91 (1947). Tradução para o inglês em "Collected Papers Of L. D. Landau", editado por D. Ter Haar (Pergamon Press, Oxford, 1965), p. 466.

[23] D.G. Henshaw and A.D.B. Woods, Phys. Rev. 121, 1266 (1961).

[24] D.D. Osheroff, R.C. Richardson and D.M. Lee, Phys. Rev. Lett. 28, 885 (1972).

[25] R.P. Feynman, Progr. Low Temp. Phys. 1, 17 (1955).

[26] R.J. Donnelly, Phys. Today 62, 34 (2009).

[27] L. Landau, Phys. Rev. 75, 884 (1949).

[28] L. Tisza, Phys. Rev. 75, 885 (1949).

[29] L. M. Brown (ed) "Selected Papers of Richard Feynman with Commentary" (World Scientific, Singapore, 2003).

[30] R.P. Feynman, Phys. Rev. 91, 1291 (1953).

[31] J.D. Vianna, Rev. Bras. Ens. Fis 40 (2018) unpublished.

[32] R.P. Feynman, Phys. Rev. 91, 1301 (1953).

[33] R.P. Feynman, Phys. Rev. 94, 262 (1954).

[34] R.P. Feynman and M. Cohen, Phys. Rev. 102, 1189 (1956).

[35] M. Cohen and R.P. Feynman, Phys. Rev. 107, 13 (1957).

[36] V.L. Ginzburg, Phys. Today 42, 54 (1989).

[37] D. Goodstein and J. Goodstein, Phys. Perspect. 2, 30 (2000).

[38] R.P. Feynman, Progress in Low Temperature Physics v. 2, (North-Holland, New York, 1955), p. 17.

[39] L. Onsager, Nuovo Cimento 6, 279 (1949).

[40] J.R. Donnelly, Quantized Vortices in Helium II (Cambridge University Press, Cambridge, 1991), p. 48.

[41] G.E. Volovik The Universe in a Helium Droplet (Claredon Press, Oxford, 2003).

[42] J. Leite Lopes, Ciência \& Sociedade no. 005/88.

[43] H.K. Onnes, KNAW Proceed. 11, 168 (1908).

[44] H.K. Onnes, Commun. Phys. Lab. Univ. Leiden 122b (1911).

[45] S. Reif-Acherman, Rev. Bras. Ensino Fís. 33, 1 (2011).

[46] J. Bardeen, L.N. Cooper and J.R. Schrieffer, Phys. Rev. 108, 1175 (1957).

[47] F. London and H. London, Proc. Royal Soc. A 149, 71 (1935).

[48] V.L. Ginzburg and L.D. Landau, Zh. Eksp. Teor. Fiz. 20, 1064 (1950).

[49] W. Meissner and R. Ochsenfeld, Naturwissenschaften 21, 787 (1933).

[50] J N. Rjabinin and L.W. Schubnikow, Nature 135, 581 (1935).

[51] A.A. Abrikosov, Soviet Physics - JETP 5, 1174 (1957).

[52] A.A. Abrikosov, Rev. Mod. Phys. 76, 975 (2004), disponível em http://nobelprize.org/nobel_prizes/ physics/laureates/2003/abrikosov-lecture.pdf

[53] B.D. Josephson, Phys. Lett. 1, 251 (1962).

[54] R.P. Feynman, R.B. Leighton and M. Sands, The Feynman's Lectures on Physics, v. 3 (Addison-Wesley, Reading, 1965). 